\title{
TRATAMENTO DE SEMENTES COM ÁCIDO SALICÍLICO NA TOLERÂNCIA DO TRIGO AO FLUMIOXAZIN
}

\section{SEED TREATMENT WITH SALICYLIC ACID IN THE TOLERANCE OF WHEAT TO FLUMIOXAZIN}

Felipe Augusto Reis Gonçalves ${ }^{\mathrm{a} *}$, Christiane Augusta Diniz Melo ${ }^{\mathrm{b}}$, Vitor Iago Pimenta de Sousa ${ }^{\mathrm{a}}$, Igor Raylson Ferreira Vinhal ${ }^{\mathrm{a}}$, Gustavo Antonio Mendes Pereira $^{\mathrm{a}}$, Marcelo Rodrigues Reis ${ }^{\mathrm{c}}$

${ }^{a}$ Instituto de Ciências Agrárias, Universidade Federal de Viçosa Campus Rio Paranaíba, Minas Gerais, Brasil. ${ }^{b}$ Departamento de Ciências Agrárias, Universidade Federal do Triângulo Mineiro Campus Iturama, Minas Gerais, Brasil. 'Departamento de Ciências Agrárias, Universidade Federal de Viçosa Campus Rio Paranaíba, Minas Gerais, Brasil.

*Autor correspondente: felipe.a.agro@gmail.com.

\section{INFORMAÇÕES DO ARTIGO \\ Histórico do artigo:}

Recebido: 25 Abril 2018.

Aceito: 20 Março 2019.

Publicado: 08 Agosto 2019.

\section{Palavras-chave/Keywords:}

Herbicida/Herbicide.

Intoxicação/Intoxication.

Triticum aestivum L./Triticum

aestivum $\mathrm{L}$.

Protetores Químicos/Safener.

Direito Autoral: Este é um artigo de acesso aberto distribuído sob os termos da Licença Creative Commons, que permite uso, distribuição e reprodução irrestritos em qualquer meio, desde que $\mathrm{o}$ autor $\mathrm{e}$ a fonte originais sejam creditados.

\section{Citação deste artigo :}

GONÇALVES, F. A. R.; MELO, C. A. D; SOUSA, V. I. P; VINHAL, I. R. F.; PEREIRA, I. R. F; REIS, I. R. F. Tratamento de sementes com ácido salicílico na tolerância do trigo ao flumioxazin.Revista Brasileira de Herbicidas , v. 18, n. 1. 2019.

\begin{abstract}
RESUMO
O tratamento de sementes com protetores químicos é uma técnica que pode aumentar a tolerância de culturas a herbicidas, sendo já utilizada em culturas como o algodão e o arroz. Este estudo foi proposto para avaliar o efeito do tratamento de sementes com ácido salicílico sobre a tolerância do trigo ao flumioxazin. O delineamento adotado foi o inteiramente casualizado, com quatro repetições. Foi utilizado o arranjo fatorial 2 x 6 , onde o primeiro fator correspondeu a ausência ou presença do tratamento de sementes com ácido salicílico e o segundo fator às doses de flumioxazin $\left(0 ; 10 ; 20 ; 30 ; 40\right.$ e $50 \mathrm{~g} \mathrm{ha}^{-1}$ i.a.) aplicadas em préemergência da cultura. Avaliações de fitointoxicação foram realizadas aos 7, 14, 21, 28 e 35 dias após a emergência. Aos 50 dias após a semeadura foi determinados o número de perfilhos por planta, altura de perfilhos férteis, matéria seca da parte aérea e da espigueta. $\mathrm{O}$ tratamento de sementes com ácido salicílico reduziu a intoxicação das plantas de trigo aos 7 , 14 e 35 dias após a emergência. O número de perfilhos férteis não foi alterado pelos tratamentos. O aumento da dose de flumioxazin afetou negativamente as demais variáveis de crescimento avaliadas (matéria seca da parte aérea, altura de perfilhos férteis e matéria seca de espigueta), sendo que o ácido salicílico influenciou positivamente a matéria seca da espigueta. Conclui-se que o flumioxazin causa intoxicação e redução do crescimento do trigo e que o ácido salicílico é alternativa para atenuar o estresse causado pelo herbicida, aumentando a tolerância da cultura.
\end{abstract}

\begin{abstract}
The seed treatment with safeners may increase the tolerance of crops to herbicides. An experiment was conducted to evaluate the effect of seed treatment with salicylic acid on the tolerance of wheat to flumioxazin. The design was completely randomized, with four replications. The treatments were arranged in a $2 \times 6$ factorial, where the first factor corresponded to the absence or presence of seed treatment with salicylic acid and the second factor to the doses of flumioxazin $\left(0 ; 10 ; 20 ; 30 ; 40\right.$ and $50 \mathrm{~g} \mathrm{ha}^{-1}$ a.i.) in pre-emergence application. The seed treatment with salicylic acid reduced the toxicity of wheat plants to 7 , 14 and 35 days after emergence. The number of fertile tillers was not affected by the treatments. Increasing the dose of flumioxazin negatively affected the growth variables evaluated (dry matter of the aerial part, height of fertile tillers and dry matter of spikelets), and the salicylic acid positively influenced the dry matter of the spikelets. The flumioxazin causes intoxication and reduces the growth of wheat. Salicylic acid is an alternative to alleviate the stress caused by the herbicide, increasing the tolerance of the crop.
\end{abstract}




\section{Introdução}

A presença de plantas daninhas em sistemas de produção do trigo pode resultar em decréscimos no crescimento e na produtividade de grãos, especialmente, quando ocorre nos estádios iniciais de desenvolvimento (PENCKOWSKI et al., 2003; CENCI et al., 2013). O período crítico de prevenção da interferência de plantas daninhas no trigo é de 12 - 24 dias após a emergência (AGOSTINETTO et al., 2008). Assim, é necessária a adoção de métodos de controle das infestantes durante esse período, sendo que o controle químico com uso de herbicidas em pré ou pós-emergência tem sido o método mais utilizado na cultura do trigo. Atualmente existem apenas duas moléculas registradas para aplicação em préemergência na cultura do trigo, pendimentalin e 2,4-D, o que limita o controle de plantas daninhas na cultura (AGROFIT, 2019). O flumioxazin apresenta potencial de uso do no cultivo do trigo, porém, também foram relatados sintomas de intoxicação da cultura pelo herbicida.

A tolerância das culturas a herbicidas está ligada a diferentes fatores intrínsecos ou não às plantas. Esta pode ser aumentada com o uso de substâncias exógenas, denominadas de antídotos ou protetores, que diminuem a intoxicação de herbicidas nas culturas (GALON et al., 2011). O uso de ácido salicílico tem sido avaliado como alternativa para atenuar a intoxicação causada por paraquat em batata-doce (ANANIEVA et al., 2002; 2004) e por glyphosate e clethodim em milho (RADWAN, 2012; AKBULUT et al., 2015). Plantas tratadas com ácido salicílico apresentaram maiores atividades de enzimas do sistema antioxidativo como catalase, superóxido dismutase e peroxidases (ANANIEVA et al., 2002; 2004; RADWAN, 2012; AKBULUT et al., 2015).

Estudos com ácido salicílico no tratamento de sementes ainda são escassos no país, principalmente relacionando a aplicação exógena como forma de combate ao estresse causado por herbicidas. Diante do exposto, objetivou-se avaliar doses de flumioxazin e o efeito do tratamento de sementes com ácido salicílico sobre a tolerância do trigo a este herbicida.

\section{Material e Métodos}

O experimento foi instalado em casa de vegetação pertencente a Universidade Federal de Viçosa, no município de Rio Paranaíba-MG. O período de condução do experimento foi de setembro a novembro de 2014.

O solo utilizado é classificado como Latossolo Vermelho Amarelo distrófico de textura argilosa (EMBRAPA, 2013). A adubação foi realizada usando-se $1,25 \mathrm{~g} \mathrm{dm}^{-3}$ do formulado 08-30-08, com base nas exigências da cultura e na análise dos atributos químicos do solo: $\mathrm{pH}\left(\mathrm{em} \mathrm{H}_{2} \mathrm{O}\right)=6,3$; M.O.S. $=2,4 \mathrm{dag} \mathrm{dm}^{-1}$; Premanescente $=15,6 \mathrm{mg} \mathrm{L}^{-1} ; \mathrm{P}$ (Mehlich-1) $=8,2 \mathrm{mg} \mathrm{dm}^{-3}$; $\mathrm{K}^{+}=58,0 \mathrm{mg} \mathrm{dm}{ }^{-3} ; \mathrm{Ca}^{2+}=4,7 \mathrm{cmol}_{\mathrm{c}} \mathrm{dm}^{-3} ; \mathrm{Mg}^{2+}=1,0 \mathrm{cmol}_{\mathrm{c}}$ $\mathrm{dm}^{-3} ; \mathrm{Al}^{3+}=0,0 \mathrm{cmol}_{\mathrm{c}} \mathrm{dm}^{-3} ; \mathrm{H}+\mathrm{Al}=2,6 \mathrm{cmol}_{\mathrm{c}} \mathrm{dm}^{-3} ; \mathrm{T}=$ $8,45 \mathrm{cmol}_{\mathrm{c}} \mathrm{dm}^{-3} ; \mathrm{V}=69,0 \%$.

O trigo, cultivar BRS 264, foi semeado em vasos com volume de $5 \mathrm{dm}^{3}$, estas foram irrigadas diariamente de forma localizada mantendo-se a umidade a capacidade de campo. $\mathrm{O}$ delineamento experimental utilizado foi $\mathrm{o}$ inteiramente casualizado, com quatro repetições. Os tratamentos foram arranjados em esquema fatorial $2 \times 6$, com o primeiro fator correspondente ao tratamento ou não das sementes de trigo com ácido salicílico e o segundo às doses $\left(0 ; 10 ; 20 ; 30 ; 40\right.$ e $50 \mathrm{~g} \mathrm{ha}^{-1}$ i.a.) do flumioxazin (Flumyzin 500 WP, $500 \mathrm{~g} \mathrm{~kg}^{-1}$ i.a., WP, Sumitomo Chemical do Brasil Representações LTDA). As sementes tratadas com ácido salicílico ficaram imersas em solução 0,5 $\mathrm{mM}$ por 6 horas e, posteriormente, foram deixadas para secar a sombra por 30 minutos antes da semeadura segundo proposto por Deef (2007).

A aplicação do herbicida flumioxazin foi realizado após o plantio de oito sementes de trigo, utilizando-se pulverizador costal pressurizado com $\mathrm{CO}_{2}$, com pressão de 2 bar, munido de bicos de jato plano, tipo leque 095.02, posicionado à altura de $50 \mathrm{~cm}$ do alvo e volume de calda equivalente a $200 \mathrm{~L} \mathrm{ha}^{-1}$. Logo após a emergência foi realizado o desbaste, deixando-se quatro plântulas por vaso após o desbaste. Os tratos culturais foram realizados de acordo com os recomendados para a cultura, procedendo ao controle de pragas e doenças sem deixar que estes influenciassem no desenvolvimento da cultura.

Avaliações de fitointoxicação foram realizadas aos 7, 14, 21, 28 e 35 dias após a emergência, seguindo escala proposta pela SBCPD (1995), que variaram de 0 (ausência de intoxicação) a 100 (morte da planta). Aos 50 dias após a semeadura, no início da fase de frutificação do trigo, procedeu-se o desmonte do experimento, determinando-se o número de perfilhos por planta, altura de perfilhos férteis, matéria seca da parte aérea e da espigueta.

Os dados foram submetidos à análise de variância para verificar a significância entre os tratamentos. Uma vez constato efeito realizou-se análise de regressão para verificação do comportamento das características em função das doses de flumioxazin. Adotou-se o nível de $5 \%$ de significância para todas as análises.

\section{Resultados e Discussão}

De acordo com os resultados da análise de variância houve interação significativa entre os fatores uso do ácido salicílico (AS) e doses de flumioxazin para a fitointoxicação aos 7, 14 e 35 dias após a emergência (DAE) do trigo. Nas avaliações realizadas aos 21 e 28 DAE não houve efeito da interação, mas sim dos fatores isolados.

As plantas oriundas de sementes não tratadas com AS tiveram níveis de intoxicação constantes de 73,80 e $85,20 \%$ aos 7 e 14 DAE, respectivamente, independente da dose (Figuras 1A e B). Em plantas de trigo cujas sementes foram tratadas com ácido salicílico a intoxicação aumentou linearmente com o incremento da dose. Os sintomas evidenciados nas plântulas foram caracterizados por clorose foliar e redução do vigor.

Aos 21 e 28 DAE, independentemente do tratamento com AS, houve aumento linear de intoxicação com o aumento da dose (Figuras 1C e D). Na avaliação realizada aos 35 DAE as crescentes doses provocaram aumento dos 


\section{F. A. R. GONÇALVES et al.}

sintomas visuais de intoxicação no trigo, contudo, plantas que receberam tratamento com AS apresentaram maior proteção aos danos causados pelo herbicida (Figura 1E). A intoxicação das plantas de trigo pelo flumioxazin está
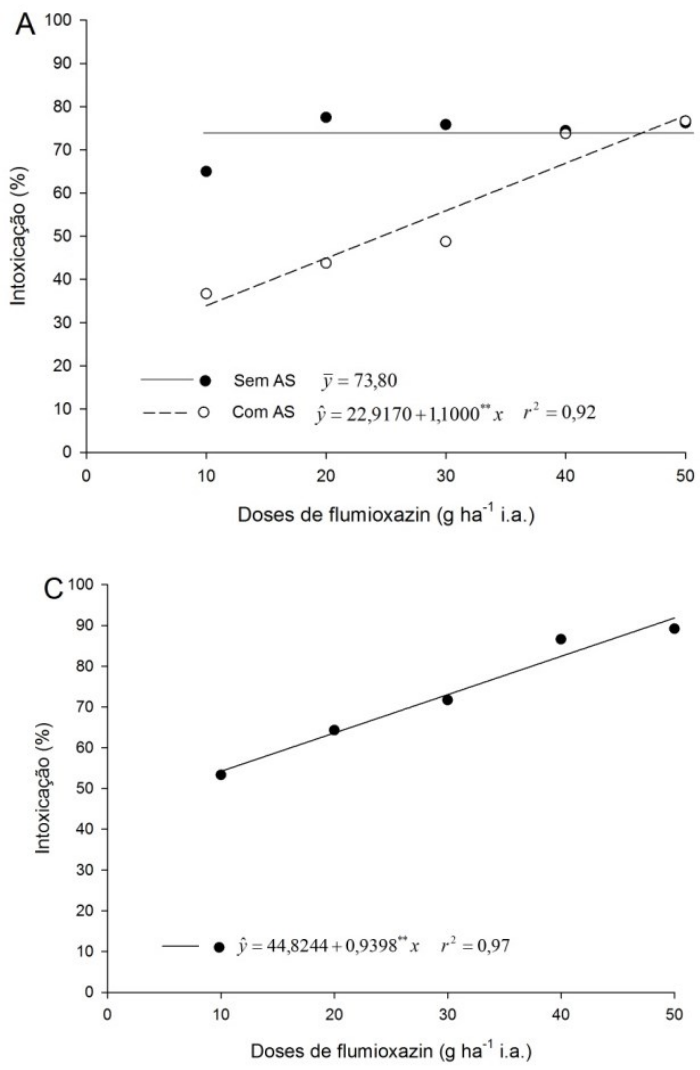

relacionada ao mecanismo de ação deste herbicida, o qual causa morte das células por extravasamento do suco celular após a peroxidação dos lipídeos da membrana plasmática.
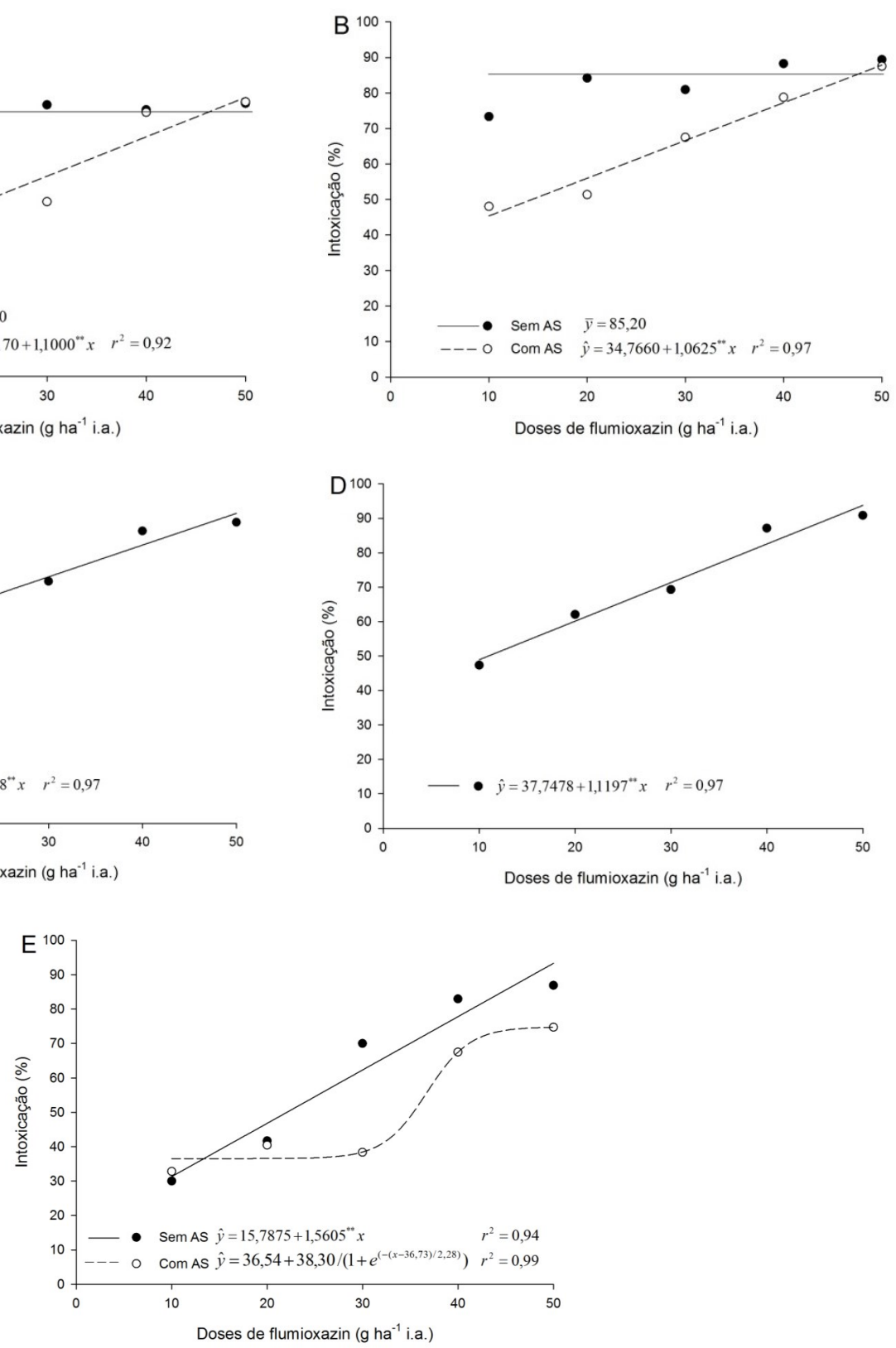

Figura 1. Intoxicação aos 7 (A), 14 (B) 21 (C), 28 (D) e 35 (E) dias após a emergência de plantas de trigo submetidas ao tratamento de sementes com ácido salicílico (AS) e cultivadas em solo que recebeu a aplicação de doses crescentes do flumioxazin em pré-emergência.

O tratamento de sementes com AS diminuiu a intoxicação das plantas de trigo em até $46 \%$, com redução aos 7 e 14 DAE nas doses de 10,20 e $30 \mathrm{~g} \mathrm{ha}^{-1}$ i.a. e aos 35 DAE nas doses de 30, 40 e $50 \mathrm{~g} \mathrm{ha}^{-1}$ i.a. (Tabela 1). Aos 21 e 28 DAE redução da intoxicação em cerca de $35 \%$ também foi verificada pelo tratamento de sementes com AS (Tabela 2). Tais resultados reforçam o papel do AS no aumento do vigor da semente e sua importância para que ocorra ótima emergência, tolerância ao estresse e crescimento uniforme das plântulas. O ácido salicílico exógeno estimula a lignificação da parede celular, direcionando as vias pentose-fosfato e chiquimato para a produção de compostos fenólicos (lignina), formando uma parede celular mais rígida e resistente aos fatores bióticos e abióticos, o que contribui para o aumento do vigor das plantas tratadas (PACHECO et al., 2007). 
F. A. R. GONÇALVES et al.

Tabela 1. Intoxicação aos 7, 14, e 35 dias após a emergência (DAE) de plantas de trigo submetidas ao tratamento de sementes com ácido salić́lico e cultivadas em solo que recebeu a aplicação de doses crescentes do flumioxazin em pré-emergência.

\begin{tabular}{|c|c|c|c|c|c|}
\hline \multirow{2}{*}{ Ácido Salicílico } & \multicolumn{5}{|c|}{ Doses de flumioxazin (g ha ${ }^{-1}$ i.a.) } \\
\hline & 10 & 20 & 30 & 40 & 50 \\
\hline & \multicolumn{5}{|c|}{7 DAE } \\
\hline Sem & $65,00 \mathrm{a}$ & $77,50 \mathrm{a}$ & $75,83 \mathrm{a}$ & $74,44 \mathrm{a}$ & $76,25 \mathrm{a}$ \\
\hline Com & $36,67 \mathrm{~b}$ & $43,75 \mathrm{~b}$ & $48,75 \mathrm{~b}$ & $73,75 \mathrm{a}$ & $76,67 \mathrm{a}$ \\
\hline \multirow[t]{2}{*}{ CV (\%) } & \multicolumn{5}{|c|}{16,80} \\
\hline & \multicolumn{5}{|c|}{$14 \mathrm{DAE}$} \\
\hline Sem & $73,33 \mathrm{a}$ & $84,17 \mathrm{a}$ & $90,83 \mathrm{a}$ & $88,24 \mathrm{a}$ & $89,38 \mathrm{a}$ \\
\hline Com & $48,06 \mathrm{~b}$ & $51,39 \mathrm{~b}$ & $67,50 \mathrm{~b}$ & $78,75 \mathrm{a}$ & $87,50 \mathrm{a}$ \\
\hline \multirow[t]{2}{*}{ CV $(\%)$} & \multicolumn{5}{|c|}{11,56} \\
\hline & \multicolumn{5}{|c|}{$35 \mathrm{DAE}$} \\
\hline Sem & $30,00 \mathrm{a}$ & $41,67 \mathrm{a}$ & $70,00 \mathrm{a}$ & 82,96 a & $86,88 \mathrm{a}$ \\
\hline Com & $32,78 \mathrm{a}$ & $40,42 \mathrm{a}$ & $38,33 \mathrm{~b}$ & $67,50 \mathrm{~b}$ & $74,72 \mathrm{~b}$ \\
\hline $\mathrm{CV}(\%)$ & \multicolumn{5}{|c|}{14,42} \\
\hline
\end{tabular}

Médias seguidas pelas mesmas letras na coluna não diferem estatisticamente pelo teste $\mathrm{F}$ à $5 \%$ de probabilidade.

Tabela 2. Intoxicação aos 21 e 28 dias após a emergência (DAE) de plantas de trigo submetidas ao tratamento de sementes com ácido salicílico e cultivadas em solo que recebeu a aplicação de doses crescentes do flumioxazin em pré-emergência.

\begin{tabular}{ccc}
\hline \multirow{2}{*}{ Ácido salicílico } & \multicolumn{2}{c}{ Épocas de avaliação } \\
\cline { 2 - 3 } & $21 \mathrm{DAE}$ & $28 \mathrm{DAE}$ \\
\hline Sem & $82,98 \mathrm{a}$ & $81,93 \mathrm{a}$ \\
Com & $63,06 \mathrm{~b}$ & $60,75 \mathrm{~b}$ \\
\hline CV $(\%)$ & 16,08 & 12,91 \\
\hline
\end{tabular}

Médias seguidas pelas mesmas letras na coluna não diferem estatisticamente pelo teste $\mathrm{F}$ à $5 \%$ de probabilidade.

Segundo Sharma e Bhardwaj (2014) o tratamento de sementes de trigo com AS, nas doses de 40 e 70 ppm, aumentou a ativação de enzimas antioxidantes, tais como superóxido dismutase, catalase, peroxidase de ascorbato e glutationa redutase, melhorando a estabilidade da membrana. Cataneo et al., (2005) observaram que a superóxido dismutase possui ação contra o estresse oxidativo induzido por oxyfluorfen, herbicida do mesmo mecanismo de ação do flumioxazin, em plantas de soja.

O flumioxazin é um herbicida inibidor da enzima PROTOX, a qual converte, por oxidação, o protoporfirinogênio IX a protoporfirina IX (RODRIGUES; ALMEIDA, 2011). Ao inibir a PROTOX ocorre acúmulo de protoporfirinogênio IX, que se difunde para o citoplasma. Há a formação de protoporfirina IX e, na presença de luz e oxigênio, espécies reativas de oxigênio são originadas, causando a peroxidação de lipídeos. Acredita-se que o tratamento de sementes com AS promova aumento da atividade de enzimas antioxidantes, as quais reduzem o estresse oxidativo causado por espécies reativas de oxigênio e, consequentemente, os sintomas visuais de fitointoxicação.

$\mathrm{O}$ número de perfilhos férteis não foi alterado pelos tratamentos, com média de 4,58 perfilhos férteis por planta de trigo. A matéria seca da parte aérea (MSPA) e a altura de perfilhos férteis (APF) não foram influenciadas pelo AS e o aumento das doses de flumioxazin causou redução linear destas. A maior dose $\left(50 \mathrm{~g} \mathrm{ha}^{-1}\right)$ reduziu a MSPA (Figura 2A) e a APF (Figura 2B) de plantas de trigo em 57,50 e $18,26 \%$, respectivamente. 

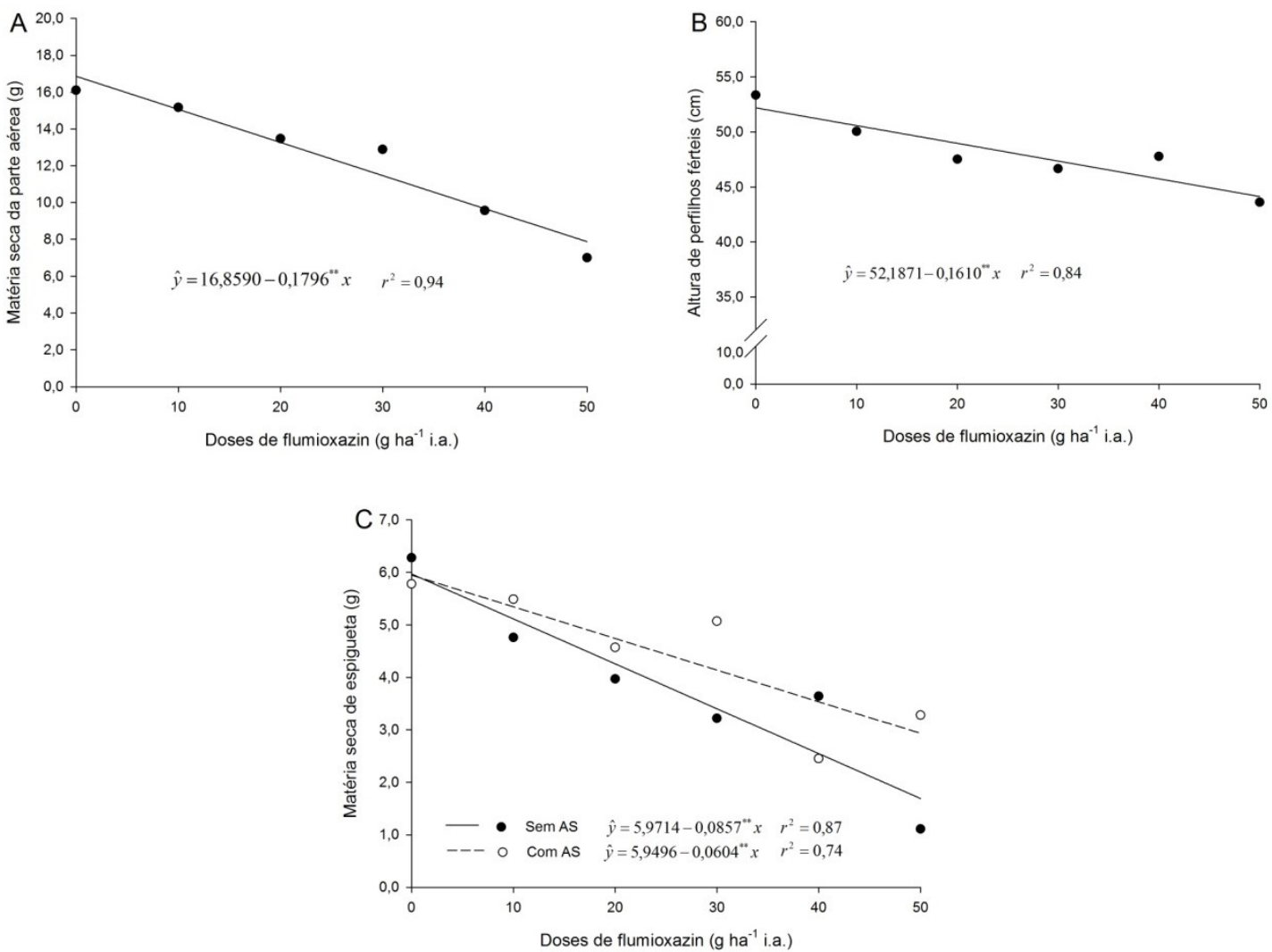

Figura 2. Matéria seca da parte aérea (A), altura de perfilhos férteis (B) e matéria seca de espiguetas (C) de trigo submetido ao tratamento de sementes com ácido salicílico (AS) e cultivado em solo que recebeu a aplicação de doses crescentes do flumioxazin em pré-emergência.

A matéria seca da espigueta (MSESP) foi afetada pela interação dos fatores com redução da variável com o incremento das doses de flumioxazin (Figura 2C). Plantas cujas sementes receberam tratamento com AS sofreram menor redução da MSESP com aumento da dose, evidenciada pela menor inclinação da reta. Plantas que não tiveram sementes tratadas acumularam 36,50 e $66,15 \%$ menos MSESP em relação às tratadas, nas doses de 30 e 50 $\mathrm{g} \mathrm{ha}^{-1}$ i.a., respectivamente (Tabela 3). Segundo McCue et al. (2000) a aplicação de reguladores vegetais às sementes pode resultar em alterações fisiológicas que podem influenciar o desenvolvimento vegetal. Tal fato pode ter contribuído para a superioridade das plantas tratadas com AS na presença do flumioxazin. O aumento de tolerância da planta de trigo ao herbicida pelo tratamento de sementes com ácido salić́lico pode atuar positivamente impedindo a intoxicação da planta, minimizando os possíveis efeitos danosos do produto sobre a produtividade da cultura em campo.

Tabela 3. Matéria seca de espiguetas de trigo submetido ao tratamento de sementes com ácido salićlico e cultivado em solo que recebeu a aplicação de doses crescentes do flumioxazin em pré-emergência.

\begin{tabular}{|c|c|c|c|c|c|c|}
\hline \multirow{2}{*}{ Ácido Salicílico } & \multicolumn{6}{|c|}{ Doses de flumioxazin (g ha ${ }^{-1}$ i.a.) } \\
\hline & 0 & 10 & 20 & 30 & 40 & 50 \\
\hline Sem & $6,28 \mathrm{a}$ & $4,76 \mathrm{a}$ & $3,97 \mathrm{a}$ & $3,22 b$ & $3,64 \mathrm{a}$ & $1,11 \mathrm{~b}$ \\
\hline Com & $5,78 \mathrm{a}$ & $5,49 \mathrm{a}$ & $4,57 \mathrm{a}$ & $5,07 \mathrm{a}$ & $2,45 \mathrm{a}$ & $3,28 \mathrm{a}$ \\
\hline CV (\%) & \multicolumn{6}{|c|}{23,14} \\
\hline
\end{tabular}

Médias seguidas pelas mesmas letras na coluna não diferem estatisticamente pelo teste $\mathrm{F}$ à $5 \%$ de probabilidade.

Estudos evidenciaram que a aplicação de AS aumentou a atividade de enzimas ligadas à tolerância de estresse abiótico no trigo (MUTLU et al., 2009; ABEDINI; HASSANI, 2015). Horváth et al. (2007) descreveram a ação e função do AS como indutor de tolerância a vários tipos de estresses abióticos, sendo importante alternativa para redução de estresse causado por herbicidas. O AS também pode ter função de acelerar a degradação do herbicida na planta, como foi observado para o herbicida isoproturon em trigo por Lu et al. (2015). A tolerância ao estresse, associada aos benefícios da aplicação exógena de ácido salićlico dão respaldo aos resultados encontrados no presente trabalho. Diante da escassez de produtos registrados para cultura do trigo e da restrita quantidade de informação na literatura brasileira acerca do assunto, a utilização deste protetor ou antídoto pode viabilizar o manejo químico de plantas 
daninhas com o uso do flumioxazin.

O uso do ácido salicílico reduziu os sintomas de intoxicação causados pelo flumioxazin ao trigo, principalmente nas menores doses do herbicida. Todavia, o crescimento da planta foi reduzido com o aumento das doses do herbicida. Esses resultados indicam potencial do uso do ácido salicílico na cultura do trigo, porém devem ser realizados outros estudos para verificar a melhor dose do protetor para maximizar os seus benefícios. Além disso, informações sobre o controle de plantas daninhas com menores doses do flumioxazin devem ser obtidas em situação de campo para validar o uso do herbicida nesse sistema.

\section{Conclusões}

As crescentes doses do flumioxazin causam aumento da fitointoxicação e promove a redução do crescimento do trigo.

O uso do ácido salicílico aumenta a tolerância da cultura, reduzindo a fitointoxicação, sobretudo até a dose de $30 \mathrm{~g} \mathrm{ha}^{-1}$ i.a., e aumentando a matéria seca de espiguetas.

\section{Referências}

Abedini, M.; Daie-Hassani, B. Salicylic acid affects wheat cultivars antioxidant system under saline and non-saline condition. Russian Journal of Plant Physiology, v.62, n.5, p.604-610, 2015.

Agostinetto, D.; Rigoli, R.P.; Schaedler, C.E.; Tironi, S.P.; Santos, L.S. Período crítico de competição de plantas daninhas com a cultura do trigo. Planta Daninha, v.26, p.271-278, 2008.

AGROFIT. Sistema de agrotóxicos fitossanitários. Disponível em <http://www.agricultura.gov.br/vegetal/ culturas/trigo $>$ Acesso em: 07 mar. 2015.

Akbulut, G.B.; Yigit, E.; Bayram, D. Investigation of the Effects of Salicylic Acid on Some Biochemical Parameters in Zea mays to Glyphosate Herbicide. Journal of Environmental \& Analytical Toxicology, v.5, p.271-279, 2015.

Ananieva, E.A.; Alexieva, V.S.; Popova, L.P. Treatment with salicylic acid decreases the effects of paraquat on photosynthesis. Plant Physiology, v.159, p.685-693, 2002.

Ananieva, E.A.; Christov, K.N.; Popova L.P. Exogenous treatment with salicylic acid leads to increased antioxidant capacity in leaves of barley plants exposed to paraquat. Plant Physiology, v.161, p.319-328, 2004.

Cataneo, A.C.; Chamma, K.L.; Ferreira, L.C.; Déstro, G.F.G.; Sousa, D.C.F. Atividade de superóxido dismutase em plantas de soja (Glycine max L.) cultivadas sob estresse oxidativo causado por herbicida. Revista Brasileira de Herbicidas, v.4, p.23-31, 2005.
Cenci, S.; Zagonel, J.; Ferreira, C.; Senger, M. Períodos de convivência entre o trigo e plantas daninhas combinados a regulador de crescimento. Revista Brasileira de Herbicidas, v.12, p.124-130, 2013.

Deef, H.E. Influence of Salicylic Acid on Stress Tolerance During Seed Germination of Triticum aestivum and Hordeum vulgare. Advances in Biological Research, v.1, p.40-48, 2007.

Empresa Brasileira de Pesquisa Agropecuária - EMBRAPA. Sistema brasileiro de classificação de solos. Brasília, DF: Embrapa Solos, 2013. 353 p.

Galon, L.; Maciel, C.D.G.; Agostinetto, D.; Concenço, G.; Moraes, P.V.D. Seletividade de herbicidas às culturas pelo uso de protetores químicos. Revista Brasileira de Herbicidas, v.10, p.291-304, 2011.

Horváth, E.; Szalai, G.; Janda, T. Induction of abiotic stress tolerance by salicylic acid signaling. Journal of Plant Growth Regulation, v.26, p.290-300, 2007.

Lu, Y.C.; Zhang, S.; Yang, H. Acceleration of the herbicide isoproturon degradation in wheat by glycosyltransferases and salicylic acid. Journal of Hazardous Materials, v,283, p.806-814, 2015.

McCue, P.; Zheng, Z.; Pinkham, J.; Shetty, K. A model for enhanced pea seedling vigour following low $\mathrm{pH}$ and salicylic acid treatments. Process Biochemistry, v.35, p.603-613, 2000.

Mutlu, S.; Atici, Ö.; Nalbantoglu, B. Effects of salicylic acid and salinity on apoplastic antioxidant enzymes in two wheat cultivars differing in salt tolerance. Biologia Plantarum, v.53, p.334-338, 2009.

Pacheco, A.C.; Custódio, C.C.; Machado Neto, N.B.; Carvalho, P.R.; Pereira, D.N.; Pacheco J.G.E. Germinação de sementes de camomila [Chamomilla recutita (L.) Rauschert] e calêndula (Calendula officinalis L.) tratadas com ácido salicílico. Revista Brasileira de Plantas Medicinais, v.9, p.61- 67, 2007.

Penckowski, L.H.; Podolan, M.J.; López-Ovejero, R.F. Influência das condições climáticas no momento da aplicação de herbicidas pós-emergentes sobre a eficácia de controle de nabiça (Raphanus raphanistrum) na cultura de trigo. Planta Daninha, v.21, p.435-442, 2003.

Radwan, D.E.M. Salicylic acid induced alleviation of oxidative stress caused by clethodim in maize (Zea mays L.) leaves. Pesticide Biochemistry and Physiology, v.102, p.182-188, 2012.

Rodrigues, B.N.; Almeida, F.S. Guia de herbicidas. $6^{\text {a }}$ ed., Londrina: Autores, 2011, 697p.

Sociedade Brasileira da Ciência das Plantas Daninhas - 
F. A. R. GONÇALVES et al.

SBCPD. Procedimentos para instalação, avaliação e análise de experimentos com herbicidas. Londrina: SBCPD, 1995. $42 \mathrm{p}$.

Sharma, A.; Bhardwaj, R.D. Effect of seed pre-treatment with varying concentrations of salicylic acid on antioxidant response of wheat seedlings. Indian Journal of Plant Physiology, v.19, p.205-209, 2014. 